Voix et Images

volxetimages

\title{
Biographies avec ou sans événements
}

\section{François Paré}

Volume 29, numéro 2 (86), hiver 2004

Le laboratoire de l'écriture : manuscrits et variantes

URI : https://id.erudit.org/iderudit/008778ar

DOI : https://doi.org/10.7202/008778ar

Aller au sommaire du numéro

Éditeur(s)

Université du Québec à Montréal

ISSN

0318-9201 (imprimé)

1705-933X (numérique)

Découvrir la revue

Citer cet article

Paré, F. (2004). Biographies avec ou sans événements. Voix et Images, 29(2),

159-162. https://doi.org/10.7202/008778ar d'utilisation que vous pouvez consulter en ligne.

https://apropos.erudit.org/fr/usagers/politique-dutilisation/ 


\section{E S S A I / É T U D ES}

Biographies avec ou sans événements

$$
+++
$$

\section{FRANÇOIS PARÉ}

Université de Waterloo

Il se dégage parfois de certains livres un fort sentiment d'intégrité intellectuelle. Cartes sur table, mots à découvert, voilà que ces livres ouverts sur le pupitre de travail dégagent une espèce de vulnérabilité rassurante. Pour beaucoup d'entre nous, la poésie joue depuis longtemps ce rôle tutélaire. Ne veille-t-elle pas depuis les premiers gestes d'écriture sur le monde et sur son histoire? Gaston Miron nous l'a dit: le poète est avant tout un compagnon, et sa marche en avant celle de tout homme et de toute femme "de bien». Et avant lui, Saint-Denys Garneau avait cherché à nous accueillir «dans la vallée spacieuse de [son] recueillement». Nous avions cheminé quelque temps à ses côtés avant de bifurquer vers d'autres lieux, d'autres refuges. "Petite étoile problématique», le poème rayonnait néanmoins sur «l'immense désert» que nous avions l'impression d'habiter.

Ce rayonnement, on le sait maintenant, n'appartient pas qu'à la poésie. En effet, la seconde moitié du vingtième siècle aura été marquée par l'émancipation fulgurante des formes multiples de l'essai. Qui n'aura pas été guidé tour à tour, depuis soixante ans, par des essayistes, tels Jean-Paul Sartre, Theodor Adorno, Simone de Beauvoir, Jacques Brault, Hélène Cixous, Michel Foucault, Fernand Dumont, Susan Sontag, Jacques Derrida, Pierre Vadeboncœur, Martin Heidegger, Jean Baudrillard et tant d'autres? Une anthologie récente, un simple manuel, dira son auteur, démontre, du reste, tout le foisonnement de ces formes au sein des pratiques littéraires contemporaines. Approches de l'essai, reprises d'études européennes et québécoises consacrées au genre même de la prose d'idées, est un ouvrage d'une richesse étonnante ${ }^{1}$. Bien que cette anthologie en format poche, préparée par François Dumont, reflète avant tout des préoccupations scolaires, sa lecture intégrale offre, comme en un dialogue improbable entre Québécois et Européens, une somptueuse réflexion sur l'écriture contemporaine en prose.

Outre une traduction d'un texte célèbre de Georg Lukács, «Nature et forme de l'essai », paru pour la première fois en français dans un numéro de la revue Études littéraires en 1972, Approches de l'essai fait place à de nombreux autres textes connus, dont «L'essai comme forme» de Theodor Adorno, «Remarques sur l'essai littéraire» de Marc Angenot, «Petite essayistique» d'André Belleau et «L'essai ou le pouvoir des 
mythes», chapitre d'un livre important de Jean Terrasse, Rhétorique de l'essai littéraire, paru au Québec à la fin des années soixante-dix ${ }^{2}$. Ce qui fait l'originalité de cette anthologie, c'est la place plus qu'équitable accordée aux essayistes québécois. Car on aurait pu imaginer une anthologie entièrement consacrée aux grands noms de la pensée française et allemande sur cette question. S'il est clair que les perspectives d'André Belleau, de Jean Terrasse ou de Marc Angenot sur l'essai ne peuvent pas être dissociées de la lecture de leurs prédécesseurs européens, nous découvrons aussi que l'essayistique québécoise fait appel à des sources plus éclectiques qui vont des premiers textes de Georges Duhamel et de Jean Éthier-Blais aux études plus récentes de Marc Angenot luimême et de Robert Vigneault sur la prose d'idée et le pamphlet. Cette curieuse anthologie produit, on le voit, un dialogue lumineux entre des auteurs jusqu'alors sans rapports explicites.

Au Québec, comme ailleurs, la pratique de l'essai s'inscrit dans une profonde recherche de la liberté. Et cette liberté prend la forme d'une mise à l'écart stratégique de l'événementiel. «L'essai est une biographie sans événement», conclut d'ailleurs Jean Marcel dans un texte sur la littérature espagnole, reproduit dans ces pages. Ainsi, l'essai permet de construire le sujet en tant que pure disponibilité dans le langage. Ce qui intéresse l'essayiste, c'est plutôt l'avènement, la naissance et le déploiement d'une subjectivité, non pas hors de l'histoire, mais hors de son histoire, libre de se raconter par des voies détournées. La sélection de textes que nous offre François Dumont dans Approches de l'essai confirme l'importance des formes décloisonnées de la pensée théorique, hors desquelles la notion de sujet ne peut plus être imaginée à l'heure actuelle.

L'essai récent de Jean-Marie Poupart, J'écris tout le temps, par besoin, par plaisir, par passion, semble à première vue répondre à de telles préoccupations ${ }^{3}$. De tous les essayistes, c'est sans doute Montaigne qui inspire à la fois la forme et le contenu de cette œuvre, bourrée de tensions et de contradictions. Chez Poupart, l'essai naît d'un besoin de se justifier. Devant le sentiment de ne pas avoir été compris, ni apprécié à sa juste mesure, l'écrivain entreprend de nous convaincre du bien-fondé de son œuvre et des idéaux qui la nourrissent. Si le temps n'est plus aux bravades de la jeunesse, c'est qu'il faut aujourd'hui dénoncer le déclin des valeurs intellectuelles dans lequel le monde semble s'être engagé. Certes, l'écrivain reconnaît ses anciennes incartades: «Tandis que je militais pour l'abolition des conventions en matière stylistique, je me gargarisais, toute ironie bue, des théories en vogue dans les couloirs de l'université» (25). Mais Poupart prendra dorénavant pour cible l'université même et son discours qu'il juge détaché du réel. Il n'aura cure d'une écriture au second degré qui ne peut être qu'atrophiée et désincarnée. Son geste à lui est urgent. Sa parole se déroule "à bâtons rompus», comme celle de Montaigne: ce que l'essayiste déteste par-dessus tout, c'est la «gloutonnerie molle, poisseuse» (53) de ceux qui restent prostrés dans la contemplation stérile de leur vie passée et des écrits des autres.

$$
+++
$$

2 Jean Terrasse, Rhétorique de l'essai littéraire, Québec, Presses de l'Université du Québec, 1977. 3 Jean-Marie Poupart, J'écris tout le temps, par besoin, par plaisir, par passion, Montréal, Leméac, coll. «L'écritoire», 2003, 154 p. 
Revenant sur plusieurs de ses romans, Poupart tient à corriger l'impression laissée par certains critiques: «Avec l'âge, j'espère avoir acquis une crédibilité qui me met à l'abri de telles remontrances.» (42) N'empêche que l'essai autobiographique qu'il nous livre aujourd'hui sombre volontiers dans la diatribe revancharde. Dans l'optique de Poupart, les critiques sont littéralement des désœuvrés: «Réitérant inlassablement les mêmes paradigmes, les mêmes devises, les mêmes figures, ils se refusent le droit de penser le monde en leurs mots. Ils finissent de la sorte par oblitérer leurs instincts les plus élémentaires.» (109) Ces idées conventionnelles - qui ne les a pas entendues lors d'un quelconque colloque? - font heureusement place à des phrases lumineuses, des lueurs de vulnérabilité dans un magma de fanfaronnades et de fausses excuses. Lorsque l'écrivain a la patience de s'attarder à l'acte même de la création, il se laisse absorber par une certaine logique contemplative dont il croyait s'être départi. Ce sont ces temps d'arrêt, alors que les phrases se bousculent, qui font la valeur de l'essai de Poupart et qui nous aident à comprendre la démarche dans laquelle il s'est engagé: «À l'origine de l'acte d'écrire, il y a toujours une révélation». L'œuvre est alors nourrie par la nostalgie de «ces atomes d'éternité échappés de la spirale de l'éphémère» (149). De tels moments de grâce sont malheureusement trop rares. "Les mauvais essayistes, écrit Poupart au dernier chapitre de son livre, chargent leur texte d'une énergie qui me semble disproportionnée par rapport aux sujets qu'ils traitent. Leurs arguments déferlent sur moi comme un mascaret. Ils me submergent, m'engloutissent» (138). J'écris tout le temps cherche, avec une affectation obsessionnelle, à produire un sujet-écrivain, comme si l'œuvre qu'il avait autrefois signée ne suffisait plus à en assurer l'existence et la légitimité. Hélas, cette statuaire, factice et outrancière, croule sous le poids accumulé des mots. On a peine à trouver ce qu'il en reste, une fois la dernière page tournée.

De tous les essayistes québécois de la seconde moitié du vingtième siècle, il me semble qu'il faut compter Hubert Aquin parmi les plus grands. La lecture récente de sa «Fatigue culturelle du Canada français» m'en a convaincu plus que jamais, tant ces paragraphes sont au cœur d'une pensée originale du colonialisme culturel et de la minorisation. La parution récente du livre de Richard Dubois, Hubert Aquin Blues ${ }^{4}$, m'avait donc fortement sollicité, d'autant plus que l'éditeur promettait un véritable «dépoussiérage» de l'image surfaite d'Aquin dans notre littérature. Il me semblait que nous étions tous prêts pour une telle remise en cause, avec laquelle Aquin lui-même, par autodérision, aurait sûrement été d'accord. Mais l'éléphant n'aura accouché que d'une souris. L'ouvrage de Dubois, anecdotique et superficiel, ne fait pas le poids. Pourtant, à l'insu de son auteur, ce livre étrange a suscité chez moi de profondes interrogations sur la nature même des rapports entre l'essayiste et son objet et sur la nécessité d'une éthique de la recherche en études littéraires.

Hubert Aquin Blues est, de l'avis de son auteur, une tentative de déstabiliser «ce $\mathrm{qu}^{\prime}$ on pourrait appeler le dispositif socio-politique mis en place au service de la geste aquinienne» (19). Pour y arriver, Dubois s'en prend non seulement à l'œuvre elle-même, engluée par une «sinistre couche de vernis» (41), mais à toute l'époque de la Révolution 
tranquille devant laquelle le critique ressent un malaise gênant: «Reconnaissons donc une première encoche à "l'amour" que l'on peut porter à l'auteur Hubert Aquin : il est daté, son époque est révolue.» (161) C'est pourquoi ce qui doit être un ouvrage sur Aquin, l'homme et l'œuvre, devient à divers moments une dérive hargneuse et sans fondement contre la génération de l'écrivain, contre les «faiseurs de mythes» qui hantent les universités québécoises, contre Aquin lui-même enfin, puisqu'il n’a fait que jouer le jeu de ses contemporains. Dès les premières lignes, Dubois s'embarque donc dans une assez curieuse croisade contre une institution littéraire québécoise qu'il accuse d'être vouée au culte du héros et à la promotion de lubies nationalistes.

À vrai dire, Richard Dubois n'est pas le premier à remettre en question la validité du référent obligé à la Révolution tranquille dans toute histoire récente du Québec. Le travail qu'il effectue ici sur Aquin en tant que lecteur des œuvres de Mauriac, Claudel, Thérèse d'Avila, Maritain et tant d'autres tenants de la France traditionaliste révèle les contradictions de toute une génération formée par l'enseignement classique. Dubois a également raison de porter son attention sur les phénomènes institutionnels qui, fortement intégrés au projet nationaliste, ont permis de définir la tradition littéraire québécoise et d'en écarter résolument les dissonances. De son vivant, Aquin n'a d'ailleurs pas cherché à se distancier de cette alliance stratégique que Dubois juge trompeuse. Il me semble, au contraire, qu'elle est fondamentale à toute lecture de son œuvre.

Dubois se sert abondamment du journal personnel d'Aquin, qu'il lit le plus souvent au premier degré. Il suit pas à pas la vie de l'écrivain, telle qu'elle s'est construite, des études collégiales à Montréal jusqu'au suicide spectaculaire, dont il voit déjà des traces dans certains textes du journal de 1964. Aquin lui semble un homme erratique, un intellectuel bourré de contradictions, un être mensonger, penchant vers l'autoflagellation, la fumisterie et la ruse. À l'image du révolutionnaire, évoquée dans Prochain épisode, Dubois oppose celle d'un écrivain, certes étonnamment ouvert au monde, mais du même souffle marqué à jamais par l'idéologie petite-bourgeoise qu'il partageait avec tous ses contemporains. Dans certains chapitres de Hubert Aquin Blues, le sarcasme et l'amertume sont tels qu'on en vient à se demander pourquoi le critique consacre tant d'énergie à "démolir» un écrivain qu'il nous dit pourtant admirer.

Il m'est resté de la lecture de cet ouvrage un surprenant sentiment d'injustice. Je suis le premier à reconnaître la nécessité de revoir les mythes socio-politiques que l'institution littéraire engendre. Cependant, quelle éthique régit le travail du critique qui s'en prend à la mémoire de l'écrivain? Que le journal d'Aquin évoque, par exemple, son désir sexuel pour les femmes plus jeunes, que le personnage de L'antiphonaire exprime de troublants fantasmes de viol, cela fait-il de l'écrivain «un violeur» qu'il faudrait juger sur la place publique, posthumément, comme si l'œuvre n'était plus que pièce à conviction dans un procès larvé contre toute une époque? Je ne le crois pas. Ce qui frappe à la lecture de l'ouvrage de Richard Dubois, c'est jusqu'à quel point, dans son désir de créer de l'événementiel à partir du littéraire, il s'écarte de la noblesse même de l'essai dont le sens, selon Lukács, émerge de sa forme et de son enracinement dans la culture. 